\title{
CORRELATION OF STATIC BALANCE WITH KNEE PROPRIOCEPTION IN ELDERLY
}

\author{
Varsha. Solanki ${ }^{* 1}$, Saraswati. Iyer ${ }^{2}$.
}

${ }^{* 1}$ Post-graduate Research Student, P.T. School and Centre, Seth Gordhandas Sunderdas Medical College \& King Edward Memorial Hospital, Parel, Mumbai, India.

${ }^{2}$ Professor and Head of Department, P.T. School and Centre, Seth Gordhandas Sunderdas Medical College and King Edward Memorial Hospital, Parel, Mumbai, India.

\section{ABSTRACT}

Background: Balance in the human body is maintained by the coordinated integration of sensory input from somatosensory, vestibular and visual system. This coordinated response to stimuli must be transmitted to appropriate muscles to maintain or to correct balance. Both static and dynamic stabilizers provide support to the normal knee joint where static stabilizers include ligament, meniscus and joint capsule which provide stabilization to the joint. Altered response in these sensory inputs may affect balance. Proprioceptive feedback is received from the sensory receptors present in the joint to maintain knee joint position, which gets altered due to microtrauma in the aging process, which thus affects balance. In elderly, this loss of balance is correlated with increased risk of falls. So, it is important to study the correlation between static balance and knee proprioception in elderly.

Purpose of the study: To understand the correlation of static balance with knee proprioception.

Methodology: It was an Observational cross- sectional study in which 70 subjects were enrolled as per the inclusion and exclusion criteria, after which a written informed consent was taken. Knee proprioception error was assessed in standing position using a goniometer. Static Balance was assessed using Tandem Stance Time. Results: The study showed negative correlation between Right knee proprioception error and tandem stance time, $r=-0.1207$ and left knee proprioception error and tandem stance time, $r=-0.1222$.

Conclusion: Thus, the study concluded that there is negative correlation between knee proprioception error and static balance, i.e. lesser the knee proprioception error better is the static balance.

KEY WORDS: Static Balance, Knee proprioception error, Tandem Stance Time.

Address for correspondence: Dr. Varsha. Solanki (PT), P.T. School and Centre, Seth Gordhandas Sunderdas Medical College \& King Edward Memorial Hospital, Parel, Mumbai-400 012, India. E-Mail: varshasolanki.vs18@gmail.com

\section{Quick Response code}

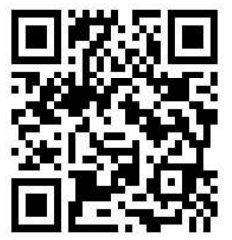

DOI: $10.16965 /$ ijpr.2020.105

International Journal of Physiotherapy and Research ISSN (E) 2321-1822 | ISSN (P) 2321-8975

https://www.ijmhr.org/ijpr.html DOI-Prefix: https://dx.doi.org/10.16965/ijpr

\section{Article Information}

Received: 12 Feb 2020

Peer Review: 12 Feb 2020

Revised: 13 Feb 2020
Accepted: 11 Mar 2020

Published (O): 11 Apr 2020

Published (P): 11 Apr 2020

\section{INTRODUCTION}

Knee joint is the most injured joint in the body. It is a complex joint which is composed of two distinct articulations which are located within a single joint capsule; Tibiofemoral and Patello femoral joint. The tibiofemoral joint is the articulation between distal femur and tibia and is a double condyloid joint with three degrees of freedom of angular motion. Flexion and extension occurring in sagittal plane around a coronal axis through the epicondyles of distal femur, medial-lateral rotation occur in transverse plane about a longitudinal axis through the lateral side of medial condyle and abductionadduction occur in frontal plane around an anterior posterior axis. The knee joint capsule and the associated ligaments restrict excessive joint motion to maintain joint integrity and 
maintain normal function. The joint capsule also provides a tight seal to lubricate the synovial fluid within the joint space. Anterior cruciate ligament $(A C L)$, Posterior cruciate ligament $(\mathrm{PCL})$, Medial Collateral ligament (MCL), Lateral collateral ligament (LCL) are the ligaments that provide support to the knee joint. These ligaments prevent excessive knee extension, varus and valgus stresses at the knee, anterior or posterior displacement of the tibia beneath femur, medial and lateral rotation of the tibia and also maintains its rotary stabilization. In normal gait, a coordinated motion between hip-knee-ankle is required. Adequate range of motion at these joints brings about the normal gait. At hip, $20^{\circ}$ of extension to $20^{\circ}$ of flexion; at knee, $0^{\circ}-60^{\circ}$ flexion and at ankle, $25^{\circ}$ plantarflexion to $7^{\circ}$ of dorsiflexion is required $[1,2]$.

Balance is the ability to maintain posture for performing activities and counteract conflicts and in other words maintaining body mass center within base of support (BOS). Balance is a complex process. Upright posture is achieved by the integration of sensory input and by planning and execution of movement. Static balance is the ability to maintain the center of gravity (COG) within the BOS and keep the center of pressure (COP) of the body as immobile as possible within the BOS. Various systems co-ordinate to maintain balance $[3,4]$. First physiological component of Sensory data, which is critical to balance, is provided primarily from visual, vestibular and somatosensory systems. Vision helps to orient the body in space by referencing vertical and horizontal axis of object around them. Acuity refers to the ability to detect subtle differences in shapes and letters, whereas contrast sensitivity is the ability to detect subtle differences in shading and patterns. Peripheral vision is the ability to see from the side while looking straight ahead, and depth perception is the ability to distinguish distance. The vestibular system also provides key sensory data for balance control by providing the Central Nervous System (CNS) with information to maintain head and body position. Another physiological component which maintains balance is the somatosensory system $[5,6]$. Proprioceptive information is provided to the CNS by the joint, tendon, muscle receptor which gives information regarding motions of the body with respect to support surface and motion of the body segments with respect to each other. Human being is aware of the position of the limbs both in moving and in stationary conditions. In normal healthy knee, both static and dynamic stabilizers provide support. Static stabilizers include ligament, meniscus and the joint capsule. They provide stabilization to the joint. The capsulo-ligamentous structures play an important role by detecting joint position and motion. The afferent information from the receptors is processed in the CNS at one of three levels: the spinal cord (for reflexive activation); the lower and mid-brain (for automatic activation) or the cortex (for voluntary movements). This information is then combined with information from the eyes and inner ear to provide an appropriate stabilizing reaction [7].

Charles Sherrington defined proprioception in 1906 as, "The perception of joint and body movements as well as position of the body, or body segments, in space." Proprioception can also be defined as the cumulative neural input from mechanoreceptors (specialized nerve endings present in joints, capsules, ligaments, muscles, tendons and skin) to the CNS. Proprioception can be described as;

a) Sense of Position: Awareness of position of one's own limbs and the orientation of their body parts with respect to one another.

b) Sense of Movement: Ability to perceive both direction and velocity of movement.

c) Sense of Force: Ability to estimate amount of muscle force that must be exerted [3].

Proprioception can be assessed by Joint Position Sense (JPS) and sense of limb movement. These components of proprioception are necessary for smooth and coordinated movements. It also maintains normal body posture, regulates normal balance and postural control and motor learning and relearning. With aging, due to deterioration in proprioception, there is decline in motor coordination and balance abilities. The somatosensory system and specifically the proprioceptive system are critically involved in sensory control of balance. A decrease in proprioception leads to abnormal 
joint biomechanics which affects functional activity such as walking. Repeated injury occurs due to abnormal pathomechanics which leads to degenerative joint disease.

Proprioception involves central and peripheral components. At peripheral level, proprioception is based on cumulative neural response from the mechanoreceptors. Internal feedback loops transmit information between and within sensory and motor areas. Advancing age causes decline in proprioception at both central and peripheral level. At peripheral level there is affection of the muscle spindle along with deficits in processing of sensory input (myelin abnormalities, axonal atrophy and reduced nerve conduction velocity) and neuromuscular performance decline. At central level, central somatosensory pathways conductive function is affected by normal aging. Aging induces progressive loss of dendrite system in motor cortex, losses in number of neurons and receptors and neurochemical changes in the brain. Age related changes in proprioception was assessed by Kaplan et. al., using two techniques that required active movement and found that older subject had reduced proprioception as compared to younger subjects. The lower limb knee joint position sense is negatively affected by aging [8-10].

Since the decrease in proprioception affects balance, which subsequently leads to falls in the elderly, there is decrease in quality of life, permanent limitation of activity or even death due to injury. Hence, it is important to understand the relation between proprioception and static balance.

\section{METHODOLOGY}

Approval was taken from the Institutional Ethics Committee and a written informed consent was taken from all the subjects. The estimated sample size was 70 . It was an Observational Cross-sectional study which was conducted in the OPD of Physiotherapy department of a Tertiary Health Care Center. Subjects included were both male and female above the age of 60 years, who could stand independently, subjects having knee pain with VAS $=0$ (at rest) and VAS 4 (on activity) [11]. Materials used were Goniometer [12, 13], Geriatric Depression Scale
(GDS) [14], Montreal Cognition Assessment Scale (MoCA) $[15,16]$ and watch. Subjects with symptomatic cardiovascular, musculoskeletal and (or) neurological condition accounting imbalance, any surgery of the lower limbs, uncorrected visual impairments, vestibular impairments, uncontrolled Hypertension and Diabetes Mellitus, subjects with GDS score $>9$, subjects with MoCA score $<26$ and use of psychotropic drugs were excluded from the study.

Data was collected in a single test session. Subjects meeting the inclusion and exclusion criteria were selected. The following were the steps to conduct the procedure.

\section{Assessment of knee proprioception error:}

Knee proprioception error was assessed in standing position.

- A goniometer with the fulcrum was placed over the lateral aspect of knee joint and tied with Velcro straps. (Figure 1)

- The subject was asked to squat till his/her knee attained an angle of $30^{\circ}$ and then asked to maintain it for 15 seconds. (Figure 2)

- Subject was blindfolded to block visual input.

- After maintaining the position for 15 seconds, the leg was returned to $0^{\circ}$ knee extension.

- The subject was then asked to return to the position perceived as the target and report.

- Each subject was made to perform 3 trials in order to obtain a realistic sample of joint position sense accuracy.

- 3 trials were conducted and the angle error was noted.

- Average of the 3 readings and the absolute value was noted $[3,7,17,18]$.

\section{Assessment of static balance:}

- Static Balance was assessed using Tandem Stance Time.

- It was performed in the standing position with the heel of preferred foot in front of and touching the toes of the other foot. (Figure 3)

- Timing was started when the person puts one foot in front of the other and the tester removes the support.

- Timing was stopped when the person moves out of Tandem or contacts external support or when completed maximum of 30 seconds [1924]. 
Fig. 1: Starting position to assess knee joint proprioception.

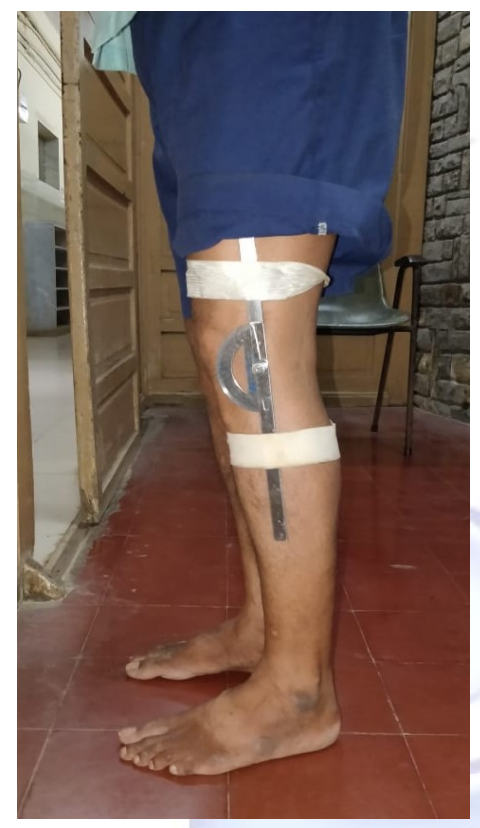

\section{RESULT}

Graph 1: Correlation of Knee joint proprioception error of Right leg at $30^{\circ}$ with static balance.

Knee proprioception error at $30^{\circ}$ (right) vs static balance

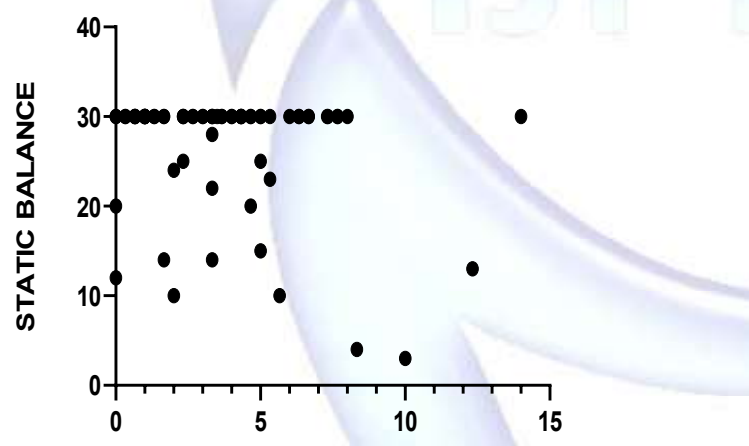

KNEE PROPRIOCEPTION ERROR OF RIGHT LEG AT $30^{\circ}$

Graph 2: Correlation of Knee joint proprioception error of Left leg at $30^{\circ}$ with static balance.

Knee proprioception error at $30^{\circ}$ (left) vs static balance

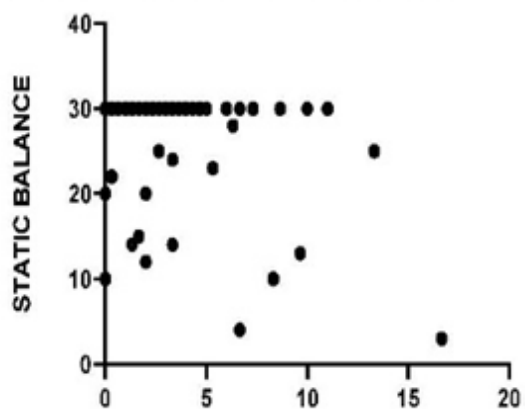

KNEE PROPRIOCEPTION ERROR OF LEFT LEG AT $30^{\circ}$

Data was analysed using Graph Pad prism version 8.1.1. Since, the data did not pass normality, Non- parametric test, i.e. Spearman
Fig. 3: Assessment of static balance using Tandem Stance Time

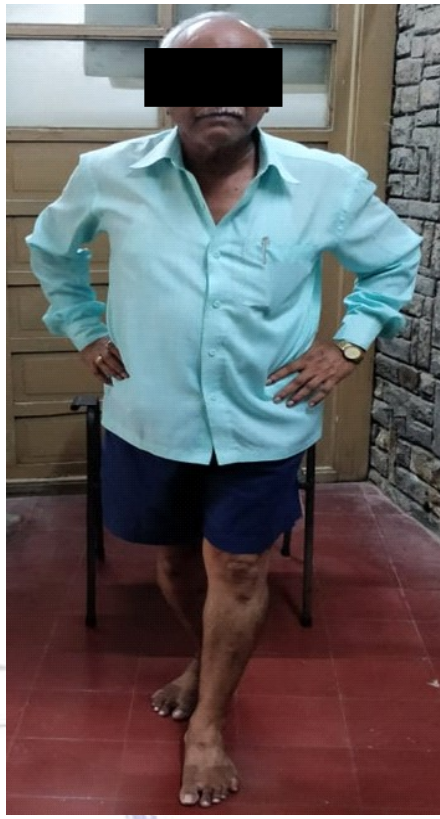

Correlation co-efficient was used for correlation between Right knee proprioception error vs Tandem stance time and Left knee proprioception error vs Tandem stance time.

A weak negative correlation of -0.1207 was found between Knee joint proprioception error of Right leg at $30^{\circ}$ with static balance. (Graph 1)

A weak negative correlation of -0.1222 was found between Knee joint proprioception error of Left leg at $30^{\circ}$ with static balance. (Graph 2)

\section{DISCUSSION}

The study shows negative correlation between knee proprioception error and static balance which means that community dwelling elderly showed better static balance with reduced knee proprioception error.

R. Bhaktiari stated that co-ordination of various sensory inputs such as the visual, somatosensory and vestibular system helps to maintain balance. Each of these systems play a significant role as it relays the information about body position and motion to the CNS, and thus it maintains balance control $[3,4]$. An ecological approach, used by Stoffregen and Riccio, described the use of sensory systems for balance control. An Intermodal theory was explained which suggested that the interaction of these systems is critically important to maintain postural control. A contrasting theory 
to the intermodal theory is the sensory reweighing theory, which suggested that even with altered sensory environment, the postural control system will be able to maintain its stance. These sensory systems contribute in a unique way to maintain postural control. It is also stated that the postural response in different sensory conditions are due to changes in the weights [6]. Sensory weighing implies that the "gain" of the sensory input depends on the accuracy of the sensory system, since it acts as a reference system for body motion. With aging, the relative accuracy of these sensory signals reduces, thus older adults rely more on vision other than vestibular and somatosensory system to maintain postural control. Peterka measure the size of postural sway as a function of magnitude of visual signals. With increase in the amplitude of visual signals in normal subjects' a decreased gain to visual inputs was demonstrative of adaptive sensory reweighting. There are two types of sensory reweighting: Inter-modality reweighting and intra-modality reweighting. Inter-modality reweighting means a change in the gain of sensory modality due to the magnitude change of the motion signal of the same sensory modality. Change in the gain of a sensory modality due to the magnitude change of motion signal from a different sensory modality is Intra- modality reweighting. Along with sensory reweighting, the postural movement strategies are used to maintain equilibrium. Strategies can be of two types: Feedback and feed forward control strategy. Feedback control refers to postural control that occurs in reference to sensory feedback from external perturbation. Feed forward control refers to postural response that are made in anticipation of a voluntary movement that is potentially destabilizing in order to maintain stability during movement [10,25-27].

Since, all the three systems, i.e. the somatosensory, vestibular and visual system rely on each other to maintain balance control, it is necessary to understand how it contributes for the function.

Somatosensory system: Proprioception is cumulative neural input to the CNS from specialized nerve endings called mechanoreceptors which are located in the joint, capsule, ligaments, muscle, tendons and skin [8].

Mechanoreceptors are specialized sensory receptors, that transduce the mechanical events as modulated neural signals to the CNS throughout afferent sensory pathway. Proprioception involves central and peripheral components. At peripheral level, proprioception is dependent on cumulative neural input from mechanoreceptors. The central component involves feedback loops that transmit information between and within the sensory and motor areas. With aging, proprioception declines at both central and peripheral level. At peripheral level, anatomical and physiological changes occur at the mechanoreceptors. Muscle spindle function changes due to decrease in the static and dynamic sensitivities and decreasing the total number of intrafusal muscle fibres and nuclear chain per spindle. These changes in the muscle spindle function contribute to reduction in proprioception. Further, there is deterioration in processing of the sensory input and neuromuscular function. At the central level, the somatosensory pathways that conduct information declines with aging. Also, with aging, there is progressive loss of the dendrite system in the motor cortex, loss in the number of neurons and neurochemical changes in the brain $[8,28]$.

Multiple studies have been conducted to study the effect of aging on proprioception. A study conducted by Skinner et. al., found that older subjects had poorer proprioception as compared to young subjects when knee proprioception was assessed under passive movement. Also, Kaplan et.al. assessed the age-related changes in proprioception requiring to perform active movement (ipsilateral and contralateral active repositioning) and found that young subjects had better perception of proprioception then elderly. Several studies showed a relationship between aging and decline in joint position sense and movement detection threshold. The lower limb knee joint position sense and ankle joint position sense are negatively affected by aging. It is also found that proprioception may be influenced by level of regular physical activity. Barrack et.al conducted a study on knee joint proprioception, concluded that the young members of the professional ballet company had significant better threshold of perception 
of joint movement than a healthy, active age-matched control group. Patrella et.al. designed a study to investigate knee joint proprioception among young volunteers and active and sedentary volunteers. Knee joint proprioception was measured at static knee angles using an electrogoniometer. 16 young subjects (19-27 years) and 24 elderly (60-86 years) were included in the study.

The elderly group was further divided into active and sedentary groups based on the level of activity. The study showed reduction in proprioception in sedentary elderly and also concluded that regular physical activity may not lead to reduction in proprioception. In a study of perception of passive movement in 60 normal control subjects, the perceived movement was better in young as compared to elderly. Kokmen et.al. found that there was decline in joint motion sensation with aging in normal subjects from 61-84 years. This reduction in proprioception may be due to structural changes in the muscle spindle, reduction in the number of intrafusal muscle fibres and nuclear chain fibres per spindle and increase in capsular thickness. With aging, there is $30 \%$ reduction in vibration induced inhibition of the stretch reflex. Muscle force production and control may be affected with alteration in motor unit function. This lack of control also has repercussion in proprioceptive ability. This shows that as the proprioception reduces, the risk of fall also increases which can also lead to fracture in elderly $[8,28,29]$.

The study shows negative correlation between knee proprioception error and static balance in elderly. The correlation was weak and could be attributed not only on somatosensory system which is discussed above but also to the vestibular, visual and musculoskeletal system also which are equally responsible for balance.

Vestibular system: Co-ordinated balance activity is maintained by the vestibular system. It provides the information about head position and movement of head with gravity to the Central Nervous system. The complex structure of the vestibular system consists of fluid filled tubes and chambers that constitutes part of the inner ear. Specialized nerve inside these structures detect the position and movement and also detect the direction of gravity. They send signals to the brain to maintain balance control in standing. The primary function of the peripheral vestibular system includes:

- Stabilization of visual image on fovea of retina during head movements to allow clear vision.

- Maintenance of postural stability during movement of head.

- Providing information used for spatial orientation [30].

Vestibular system sends information to the oculomotor nucleus which then triggers the reflexes such as Vestibulo Ocular Reflex (VOR) and Vestibulo Spinal Reflex (VSR). They act to stabilize the visual field by stabilizing the eyes and helps to maintain postural stability during stance (static balance). With aging, decline in VOR stability, affects their balance during stance. It is assumed that subtle age-related changes in VOR even if it is not causing dizziness and other vestibular symptoms can affect gaze, stability and gaze fixation activity in elderly, thus eventually affecting balance, gait and postural instability. By 70 years of age, there is $40 \%$ loss of vestibular hair and nerve cells. One of the functions of the vestibular system is that of an absolute reference system which plays an important role in situations of visual and somatosensory conflict. With aging the absolute system tends to decline. Since, it acts as an absolute reference system, vestibular input contributes to amplitude of automatic postural adjustments to balance threats. Thus, older adult with vestibular deficit would show postural responses that are inappropriately small [31-34].

Visual system: With aging, elderly tend to rely more on visual input as compared to other sensory systems. This reliance upon vision, places older adults at risk for falls because they are often not aware of the magnitude of their body motion [27].

It is estimated that after the $6^{\text {th }}$ decade, many people tend to have a rapid decrease in vision. Maki and Mc Elroy hypothesized that as age advances, some individual may rely more on vision than on vestibular or proprioceptive information for balance. A study conducted on 136 participants aged 59-97 years, found that 
$43 \%$ of the participants who had experienced one or more falls in the past were significantly relying on visual field on visual function tests than the other $57 \%$ of participants who had not fallen [35]. Study conducted by Karger, was to determine the influence of stationary anchors and vection (illusion of self-motion) upon egomotion (environmental displacement) in young, young-old and old adult group using the moving room paradigm which showed that in all optical flow conditions, old adult produced more postural motion than young old adults. Youngadult rated their vection higher, yet produced significantly less COP displacement than other older adult. There was decrease in postural responses when portion of the visual scene was occluded in central or peripheral level which indicate visual sensitivity to visual scene motion when information is available to all the areas of the retina. Static image in the visual scene provide a reference point which was found to assist in stabilization thus producing decreased risk of fall [36]. A Study conducted by Sundermeiser et.al. compared the effects of visual flow on postural responses of young stable and unstable older adults, where it showed that the unstable older adult showed significantly more reliance on visual flow than the young or stable adults as measured by COP. It was also noticed that unstable older adults had used higher level of sheer forces when compensating for simulating postural sway, thus suggesting greater use of hip strategies even in response to visual perturbations to posture [31, 37].

The Beaver Darn eye study found that consistent relationship exists between falls and hip fractures and visual function. The visual function tests were best corrected visual acuity, current binocular activity, near acuity, contrast sensitivity and visual threshold to light. The authors found that subjects that were more than 60 years of age and classified as having poorer best corrected acuity, decreased current binocular activity, poor nearer acuity and increased visual sensitivity to light were more likely to have two or more falls [27]. Also, a study was conducted to compare Eyes open vs Eyes closed balance training showed that there was no difference with eyes open and eyes closed training $[35,38]$.
Musculoskeletal system: The skeletal musculature, skeleton and its joints constitutes the motor apparatus of the balance control system. From the mid- twenties, there is progressive loss of lean body mass, such that the cross- sectional area of the vastus lateralis, decreases by approximately $40 \%$ between $20-80$ years of age. Between the $5^{\text {th }}-6^{\text {th }}$ decade, there is peak levels in muscle strength, after which there is accelerated decline by $50 \%$ by 80 years of age [39-41].

Muscle force output depends on contractile length as well as series elasticity. With aging there is alteration in these muscular properties which alters the neural transmission into muscular force. Many muscles contribute to postural stability, those surrounding the ankle joint are specifically important. Age related changes in the ankle joint may thus influence balance control. Age related changes in joint strength, muscle size, activation capacity and achillies tendon stiffness explains $70 \%$ variance in balance performance during postural challenges of tandem stance. Muscle such as gastrocnemius and soleus play an important role in maintaining upright posture. Age- related atrophy of the faster-contracting type -II muscle fibres causes the gastrocnemius to be weaker and slower in older adults. Reports have shown that gastrocnemius muscle in the older adults displayed altered force-velocity relationship, while soleus muscle did not show any change. Dorsiflexors and plantar flexors muscle in older adult showed age related differences in muscle morphology and muscle properties. Elderly population had small amount of contractile tissue relative to total muscle volume and displayed reduced maximal isometric force, slower force velocity relations altered force- length properties and stiffer series elasticity. The gastrocnemius and soleus stiffness were positively correlated with mean absolute COP speed during quiet stance. Elderly displayed a poor performance on static postural tests and muscle strength was strongly related to age related decrements [42-48]. Studies have found that there is a correlation between lower extremity weakness and increased risk of falls and with interventions in lower extremity strengthening, there is improvement in balance[49].

Thus, the above discussion justifies that static 
balance is not only maintained by the somatosensory system but also relies on the visual, vestibular and musculoskeletal system. Thus, it shows that there is negative correlation between knee proprioception error and Tandem stance time.

\section{CONCLUSION}

Thus, we conclude that there is negative correlation between knee proprioception error and static balance, i.e. lesser the knee proprioception error better is the static balance.

\section{Clinical implication}

According to the discussion, static balance is not only maintained by the somatosensory system but also relies on the visual, vestibular and musculoskeletal system. Static balance cannot be improved by training only one sensory system. In order, to improve balance, we need to train visual system, vestibular system and proprioceptive system.

\section{- Training visual system:}

a. Zooming with eyes.

b. Figure of ' 8 ' movement.

c. Rhythmic eye movements.

d. Directional eye movements.

\section{- Training vestibular system:}

a. Gaze stabilization exercise.

b. Head movement exercise.

c. Standing vestibular exercise.

d. Obstacle course.

e. Target and optic flow.

\section{- Training proprioceptive system:}

a. One leg stance.

b. Wobble board.

c. Tai-chi.

d. Standing on different surfaces like sand, foam.

\section{- Training musculoskeletal system:}
a. Squatting
b. Lunges
c. Thera bands \& weights.

Thus, training the subject with the above exercises will reduce knee proprioception error and increase static balance.

\section{Conflicts of interest: None}

\section{REFERENCES}

[1]. Cynthia c. Norkin, Pamela K. Levangie. Joint Structure and Function- A comprehensive Analysis. $5^{\text {th }}$ Edition, Jaypee Brothers Medical Publishers (P) Ltd,2011, p395-439

[2]. Carolyn Kisner, Lynn Allen Colby. Therapeutic Exercise- Foundation and Techniques. $6^{\text {th }}$ Edition, Jaypee Brothers Medical Publishers (P) Ltd, 2012, p765-770

[3]. Dhwani Jain, Mugdha Oberoi, Veena Krishnanand. Correlation of static and dynamic balance with knee proprioception in normals. Int J Physiother Res 2017;5(4):2215-2219

[4]. Reza Bhaktiari. Evaluation Of static and dynamic balance and knee proprioception in young professional soccer players. Annal of Biological research, 2012;3(6):2867-2873

[5]. M. Dena Gardiner: The Principles of Exercise Therapy. $4^{\text {th }}$ Edition, CBS Publishers \& Distributors Pvt. Ltd., p2-6.

[6]. Darcy. A. Umphred, Neurological Rehabilitation, $5^{\text {th }}$ edition, p732-739.

[7]. Madhavi Lokhande and Jaimala Shetye, Assessment of knee joint proprioception in weight bearing and non-weight bearing positions in normal subjects. JKIMSU 2013;2.

[8]. Fernando Ribeiro, Jose Oliveira. Aging effects on joint Proproception: the role of physical activity in proprioception preservation, Eur Rev Aging Phys Act 2007;4:71-76.

[9]. Ribeiro F, Oliveira J. Factors influencing proprioception: what do they reveal?. chapter; 2011 Sep 9.

[10]. Woollacott MH, Shumway-Cook A, Nashner LM. Aging and posture control: changes in sensory organization and muscular coordination. Int J Aging Hum Dev 1986;23(2):97-114.

[11]. Gillian Hawker, Samra Mian, et al. Measures of Adult pain, Arthritis Care and Research, November 2011;63(11):S240-S252.

[12]. Steven Milanese; Susan Gordon; School of Public Health. James Cook University, Reliability and validity of universal goniometer 2016.

[13]. Lucie Brosseua, Sonia Gringras: Intra and intertester reliability and criterion validity of the parallelogram and universal goniometers for measuring maximum active knew flexion and extension of patients with knee restrictions. Archives of physical medicine and Rehabilitation, March 2001;82(3):396-402.

[14]. Yesavage JA, Brink TL, Rose TL, Lum O, Huang V, Adey $M$, Leirer VO: Development and validation of a Geriatric Depression Screening Scale: A preliminary report. J Psychiatr Res. 1982-1983;17(1):37-49.

[15]. McLennan SN, Mathias JL, Brennan LC, Stewart S: Validity of Montreal Cognitive Assessment (MoCA) as a screening test for mild cognitive impairment $(\mathrm{MCl})$ in cardiovascular population. JGeriatr Psychiatry Neurol. 2011 March;24(1):33-8.

[16]. Valcour VG, Maaki KH, Kurb JD, Blanchette PL: The detection of dementia in primary care setting. Arch Inter Med. 2000 Oct23;160(19):2964-8. 
[17]. Pincivero DM, Bachmeier B, Coelho AJ: The effect of joint angle and reliability on knee proprioception. Med Sci Sports Exerc, 2001 Oct;33(10):1708-12.

[18]. Gökben Hýzlý Sayar, Hüseyin Ünübol Assessing proprioception- The Journal of Neurobehavioural Sciences 2017;4(1).

[19]. Assessment protocols for Standardized Balance Tests.

[20]. Jack Guralnik, Luigi Ferrucci, Eleanor Simonsick, Marcel Salive, Robert Wallace: Lower Extremity Function in persons over the age of 70 years a predictor of subsequent disability. The New England Journal of Medicine, 1995;332(9):556-561.

[21]. Guralnik, Ferrucci, Glynn, Berkman,Blazer, Scherr, Wallace (1994) A short physical performance battery assessing lower extremity function: Association with self-reported disability and prediction of morality and nursing home admission. Journal of Gerontology, 1994;49(2):M85-M92.

[22]. Tyson SF, DeSouza LH, Reliability and validity of functional balance tests post stroke. Clin Rehabil. 2004 Dec;18(8):916-923.

[23]. Shirley Ryan Abilitylab- Sharpened Romberg

[24]. Elizabeth Hile, Jennifer Brach, Subashan Perera, David Wert, Jessie M, VanSwearingen, Stephanie A, Studenski, Interpreting the need for Initial Support to perform Tandem Stance Tests of Balance: Phys Ther. 2012 Oct; 92(10): 1316-1328.

[25]. Stoffregen TA, Riccio GE, An Ecological theory of orientation and the vestibular system. Psychol Rev;Database-Psyc Articles,1988;95(1);3-14.

[26]. Kelvin S.Oie, Kim Kiemel, John J Jeka:Multisensory fusion:simultaneous reweighting of vision and touch for control of human posture, Cognitive Brain research ; 2002;14(1);164 - 176.

[27]. Liana Nafisa Saftari and Oh-Sang Kwon. Ageing vision and falls: a review, Journal of Physiological Anthropology (2018) 37:11.

[28]. Kararizou E, Manta P, Kalfakis N, Vassilopoulos D. Morphometric study of the human muscle spindle. Anal Quant Cytol Histol 2005;27(1):1-4.

[29]. Daniel J. Goble, James P. Coxon, Nicole Wenderoth, Annouchka Van Impe, Stephan P. Swinnen Proprioceptive sensibility in the elderly: Degeneration, functional consequences and plastic-adaptive processes Neuroscience and Biobehavioral Reviews 2009;33:271-278.

[30]. Susan O'Sullivan, Physical Rehabilitation, Fifth Edition, Chapter 18, Page 1000.

[31]. Marjorie Hines Woollacott Systems Contributing to Balance Disorders in Older Adults Journal of Gerontology: Medical Sciences 2000;55A(8):M424M428.

[32]. Tanu Khanna, Sandeep Singh. Effect of Gaze Stability Exercises on Balance in Elderly, IOSR Journal of Dental and Medical Sciences (IOSR-JDMS) 2014;13(9) Ver. 1:41-48.

[33]. C. Paquette, and J. Fung, Old age affects gaze and postural coordination, Gait \& Posture, 2011;33(2):227-32.

[34]. Christopher K. Zalewski Aging of the Human Vestibular System Semin Hear. 2015 Aug; 36(3):175-196.

[35]. Carpenter, Cara; Stanford, Megan; and Putnam, Katie. A Comparison of Eyes Open Versus Eyes Closed
Balance Training. School of Physical Therapy. 2001;161.

[36]. Haibach P, Slobounov S, Newell K. Egomotion and vection in young and elderly adults. Gerontology. 2009;55:637-43.

[37]. Sundermeier L, Woollacott M, Jensen J, Moore S. Postural sensitivity to visual flow in aging adults with and without balance problems. J Gerontol Med Sci. 1996;51A:M45-M52.

[38]. Klein BEK, Klein R, Lee KE,Cruickshanks KJ. Performance-based and self-assessed measure of visual function as related to history of falls, hip fractures and measures of gait time. Ophthalmology 1998 Jan;105(1):160-164.

[39]. Nnodim JO, Yung RL. Balance and its Clinical Assessment in Older Adults - A Review. J Geriatr Med Gerontol 2015;1:003.

[40]. Lexell J, Taylor CC, Sjöström M. What is the cause of the ageing atrophy? Total number, size and proportion of different fiber types studied in whole vastus lateralis muscle from 15- to 83-year-old men. J Neurol Sci 1988;84:275-294.

[41]. Larsson L, Grimby G, Karlsson J. Muscle strength and speed of movement in relation to age and muscle morphology. J Appl Physiol Respir Environ Exerc Physiol 1988; 46: 451-456.

[42]. Christopher J. Hasson, Richard E.A. van Emmerik, and Graham E. Caldwell. Balance Decrements Are Associated with Age-Related Muscle Property Changes. Journal of Applied Biomechanics, 2014;30:555-562.

[43]. Onambele GL, Narici MV, Maganaris CN. Calf muscle-tendon properties and postural balance in old age. J Appl Physiol. 2006;100(6):2048-2056.

[44]. Frey D, Schneider C, Xu L, Borg J, Spooren W, Caroni P. Early and selective loss of neuromuscular synapse subtypes with low sprouting competence in motoneuron diseases. J Neurosci. 2000;20(7):2534-2542.

[45]. Hasson CJ, Caldwell GE. Effects of age on mechanical properties of dorsiflexor and plantarflexor muscles. Ann Biomed Eng. 2012;40(5):1088-1101.

[46]. Hasson CJ, Kent-Braun JA, Caldwell GE. Contractile and non-contractile tissue volume and distribution in ankle muscles of young and older adults. J Biomech. 2011;44(12):2299-2306.

[47]. Hasson CJ, Miller RH, Caldwell GE. Contractile and elastic ankle joint muscular properties in young and older adults. P LoS ONE. 2011;6(1):e15953

[48]. Matheus M. Gomes, Júlia G. Reis, Thamires M. Neves, Marina Petrella, Daniela C.C. de Abreu Impact of Aging on Balance and Pattern of Muscle Activation in Elderly Women from Different Age Groups International Journal of Gerontology 2013;7:106-111.

[49]. Aoyama M, Suzuki Y, Kuzuya M. Muscle Strength of Lower Extremities Related to Incident Falls in Community-Dwelling Older Adults. J Gerontol Geriat Res 2015;4:207.

How to cite this article: Varsha. Solanki, Saraswati. Iyer. CORRELATION OF STATIC BALANCE WITH KNEE PROPRIOCEPTION IN ELDERLY. Int J Physiother Res 2020;8(2):33983406. DOI: 10.16965/ijpr.2020.105 